\title{
Circuit training enhances function in patients undergoing total knee arthroplasty: a retrospective cohort study
}

Wei-Hsiu Hsu ${ }^{1,2+}$, Wei-Bin Hsu' ${ }^{1+}$, Wun-Jer Shen ${ }^{3}$, Zin-Rong Lin ${ }^{4}$, Shr-Hsin Chang ${ }^{1}$ and Robert Wen-Wei Hsu ${ }^{1,2^{*}}$

\begin{abstract}
Background: The number of patients receiving total knee arthroplasty (TKA) has been rising every year due to the aging population and the obesity epidemic. Post-operative rehabilitation is important for the outcome of TKA.

Methods: A series of 34 patients who underwent primary unilateral TKA was retrospectively collected and divided into either exercise group $(n=16)$ and control group $(n=18)$. The exercise group underwent a 24-week course of circuit training beginning 3 months after total knee arthroplasty (TKA). The effect of circuit training on TKA patients in terms of motion analysis, muscle strength testing, Knee injury and Osteoarthritis Outcomes Score (KOOS) questionnaire and patient-reported outcome measurement Short-Form Health Survey (SF-36) at the pre-operation, pre-exercise, mid-exercise, and post-exercise.

Results: Motion analysis revealed the stride length, step velocity, and excursion of active knee range of motion significantly improved in the exercise group when compared to those in the control group. KOOS questionnaire showed a greater improvement in pain, $A D L$, and total scores in the exercise group. The SF-36 questionnaire revealed a significant improvement in general health, bodily pain, social function, and physical components score in the exercise group.
\end{abstract}

Conclusions: The post-operative circuit training intervention can facilitate recovery of knee function and decrease the degree of pain in the TKA and might be considered a useful adjunct rehabilitative modality. The ultimate influence of circuit training on TKA needs further a prospective randomized clinical trial study and long-term investigation.

Trial registration: NCT02928562

Keywords: Circuit training, Total knee arthroplasty, Motion analysis, KOOS, SF-36

\section{Background}

Total knee arthroplasty (TKA) is a well-accepted procedure for the treatment of advanced osteoarthritis of the knee joint [1], with good long-term survivorship of the implants and satisfactory surgical outcome [2]. The number of patients receiving TKA has been rising every year due to the aging population and the obesity

\footnotetext{
* Correspondence: wwh@cgmh.org.tw

${ }^{\dagger}$ Equal contributors

${ }^{1}$ Sports Medicine Center, Chang Gung Memorial Hospital, No. 6, West Section, Chia-Pu Road, Putz City 61363, Chiayi Country, Taiwan, Republic of China

${ }^{2}$ Department of Orthopaedic Surgery, Chang Gung Memorial Hospital, No. 6, West Section, Chia-Pu Road, Putz City 61363, Chiayi Country, Taiwan, Republic of China

Full list of author information is available at the end of the article
}

epidemic [3-5]. Enhancing the recovery process after TKAs is an important issue. Physical exercise was suggested to enhance muscular strength, muscular endurance, cardiovascular fitness, flexibility, agility, balance, and coordination in the healthy aged population [6]. Physical exercise could further increase the range of motion and quadriceps strength in TKA patients [7]. However, these effects of post-operative exercise were usually limited by a short hospital stay, impaired exercise adherence, and in compliance to exercise regimens [8-10].

A more balanced exercise approach is therefore recommended for TKA patients [11]. Circuit training comprised of different exercise principles, including stretching exercise, aerobic training, and resistant 
training. Multiple stations for training different muscle groups, stretching exercise, and aerobic exercise was employed to progress towards cardiovascular fitness and muscle strength [12]. We had previously reported its positive effect on body composition improvement in overweight women [9]. Meanwhile, good exercise adherences were also observed with concomitant increases in the mental domain of functional score, since the incidence of female osteoarthritis (OA) is twice that of men [13] and the number undergoing TKA in the female is about twice in men between 2001 and 2010 [14]. The retrospective study focused on the female and assessed the feasibility and effect of post-operative circuit training intervention on TKA. The circuit training intervention would carry out at 3 months after the operation for 6 months. The assessments of this intervention were measured using motion analysis and muscle strength testing, KOOS questionnaire, and patient-reported outcome measurement Short-Form Health Survey (SF-36). The goal was to demonstrate the effect, if any, that circuit training intervention might have on post-operative knee functional recovery and daily activities.

\section{Methods}

\section{Participants}

The aim was to demonstrate the effect, if any, that circuit training intervention might have on postoperative knee functional recovery and daily activities in female TKA.

The inclusion criteria of the study included end-stage OA and female. Patients with diabetes, neuromusculoskeletal disorders, severe chronic medical disease, history of fracture of a lower limb, artificial limb, and being otherwise unsuitable for exercise training were excluded.

From October 2013 to August 2015, a consecutive cohort of 34 patients underwent TKA (16 in the exercise group and 18 in the control group) at Chang Gung Memorial Hospital Chiayi branch was included in the current study.

The participants in the exercise group practiced a 24week circuit training intervention while the control group followed the routine post-operative rehabilitation protocol, including quadriceps training and range of motion exercise during the same time period.

\section{Intervention}

The circuit training program included stretching, aerobic training, and resistance training. The order of practice was performed in the following pattern: stretching/ aerobic training/resistance training/aerobic training/resistance training/stretching. Each exercise was performed for $10 \mathrm{~min}$, with a $30 \mathrm{~s}$ rest period between each exercise, and the program was carried out three times a week for 24 weeks. Aerobic training consisted of riding an exercise bike with the intensity of $60-80 \%$ target heart rate, and the resistance training was performed on hydraulic resistance equipment (AGOSS, Taipei, Taiwan) set at an intensity of $60-80 \%$ one repetition maximum. Six exercise machines were randomly chosen from ten exercise machines which included six types of equipment for the upper limbs and four for the lower limbs. The program was hospital-based and supervised by one exercise therapist at the Sports Medicine Center of Chang Gung Memorial Hospital Chiayi branch.

\section{Objectives}

The aim of the study was to assess the feasibility and effect of post-operative circuit training intervention on TKA women. The effect of this intervention was measured using motion analysis, muscle strength testing, Knee injury and Osteoarthritis Outcome Score (KOOS) questionnaire and Short-Form Health Survey (SF-36) measurement patient-reported outcome. The goal was to demonstrate the effect, if any, that circuit training intervention might have on post-operative knee functional recovery and daily activities.

\section{Outcomes}

All measurements in both the exercise group and control group were performed at the following time points, i.e., before TKA operation (pre-operation), before exercise (pre-exercise), at 12 weeks after the beginning of the circuit training program (mid-exercise), and after completion of the circuit training (post-exercise).

\section{Gait analysis}

Gait analysis was performed by a three-dimensional, eight-camera motion capture system (VICON, Oxford Metrics, London, England) synchronized with two force platforms (OR6, AMTI, Watertown, Massachusetts) to record ground reaction force. Marker data were sampled at $100 \mathrm{~Hz}$. Force platform data were sampled at $1000 \mathrm{~Hz}$. Data collection starts with standing calibration to identify joint centers and create a segment impeded coordinate system. After calibration, subjects practiced walking until reaching a constant self-selected speed. The collected trials fell within $5 \%$ of the practiced speed with clear contact of only one foot on each force plate. Five walking trials were collected for each subject. While kinematics and kinetics of the segments are calculated, the whole body is modeled as a segment-linkage system consisting of the head, trunk, pelvis, bilateral upper arms, forearms, hands, thighs, shanks, and feet. Reflective markers attached on the segments were used to establish coordinate systems representing each segment. Data were processed utilizing the Nexus motion analysis system (VICON; Oxford Metrics Ltd. Ver.1.6.5), which was integrated with data recording software. 


\section{Muscle strength}

Lower extremity muscle strength (including extension and flexion of the hip and knee, dorsi, and plantar flexion of the ankle) was measured using the HUMAC NORM system (CSMi, Stoughton, MA) with the concentric/eccentric contraction mode at an angular velocity of $60^{\circ}$ per second. All measurements were evaluated with the participant in a sitting position. Isokinetic tests were performed five times for each participant, and each test was separated by a rest period of $3 \mathrm{~min}$. The participants received verbal encouragement during the exertion of peak torque. The muscle strength was presented as a peak torque which was normalized to body weight [15].

\section{KOOS assessment}

Clinical knee scoring using the Chinese version KOOS scale was performed in the outpatient self-explanatory assessment [16]. The KOOS is a 42-item selfadministered knee-specific questionnaire assessing pain (9 items), symptoms (7 items), activities of daily living (ADL, 17 items), function, sports and recreational activities (sports/rec, 5 items), and knee-related quality of life (QOL, 4 items) in five separate subscales. All items are scored 0 to 4 ; for each subscale, the scores are transformed to a 0 to 100 scales ( 0 representing extreme knee problems and 100 representing no knee problems) [17].

\section{Quality of life}

Quality of life was assessed using the Short-Form Health Survey (SF-36, Taiwan version [18]). The SF-36 questionnaire is a multi-purpose and short-form health survey, which is commonly used to evaluate patients' quality of life in clinical practice. A total of eight domains were evaluated in this questionnaire including physical functioning (PF), role limitation due to physical problems (RP), bodily pain (BP), general health $(\mathrm{GH})$, vitality (VT), social functioning (SF), role limitation due to emotional problem (RE), and mental health $(\mathrm{MH})$. Additionally, the eight health domains can be used to provide physical component summary (PCS) and mental component summary (MCS) scores.

\section{Statistical analysis}

All data analysis was done using the Statistical Package for the Social Sciences, Windows version 17.0 (SPSS, Chicago, IL, USA). All continuous data are presented as the mean \pm standard deviation. Generalized estimating equations (GEEs) [19] were used for determining the differences between the exercise and control groups across the time period. A $p$ value of $<0.05$ was considered statistically significant.

\section{Results}

The demographic data, including age, height, and weight was similar in the exercise and control groups (Table 1). The mean age was 72 and 70 years old for the exercise and control groups, respectively.

In gait analysis, it was noted both exercise and control group were shown similar in gait parameters at preoperation and pre-exercise evaluation. Three months after beginning exercise interventions, the stride length for exercise and control group were $101.6 \pm 3.4$ and $85.0 \pm 5.5 \mathrm{~cm}$, respectively $(p=0.01)$. Similarly, step length and step velocity were greater in the exercise group than those in control group. $(p<0.05)$ (Table 2$)$. Meanwhile, the excursion of knee range of motion was $48.6 \pm 1.2$ and $44.5 \pm 1.6$ degree for exercise and control group, respectively $(p<0.05)$. The differences in stride length and excursion of knee range of motion (ROM) lasted till the post-exercise evaluation (Table 2). When comparison was performed within the individual group in temporal fashion, it was shown that stride length, stride velocity, step length, and step velocity increased in mid-excise assessment in the exercise group, while no such difference was noted in control group. The control group demonstrated increases in these parameters at post-exercise assessment $(p<0.05)$. Circuit training improved gait pattern at an earlier time point compared with the control group.

In isokinetic muscle strength assessment, it was shown that the maximal knee extensor torque for the exercise group was $24.4 \pm 5.2,31.4 \pm 3.3,44.0 \pm 4.8$, and $51.0 \pm 5.9 \mathrm{~N}-\mathrm{m} / \mathrm{Kg}$ for pre-operation, pre-exercise, midexercise, and post-exercise, respectively $(p<0.05)$. Similar increases were demonstrated in the control group for knee extensor (Table 3). Indeed, it was shown that maximal muscle strength in hip extensor, hip flexor, knee extensor and knee flexor, ankle plantar flexor, and dorsiflexor all increased after TKA surgery in a temporal fashion for both exercise and control group $(p<0.05)$. Meanwhile, it was shown that there were no differences between the exercise and control group at all time point (Table 3). Meanwhile, we measured the muscular strength of lower extremity since previous studies have shown that knee extensor strength is closely correlated to functional performance, especially after TKA [20, 21].

Table 1 Demography of participants

\begin{tabular}{lll}
\hline & Exercise group $(n=16)$ & Control group $(n=18)$ \\
\hline Age (years) & $72.1 \pm 6.7$ & $69.6 \pm 8.2$ \\
Height $(\mathrm{cm})$ & $152.6 \pm 5.6$ & $154.3 \pm 6.4$ \\
Weight $(\mathrm{kg})$ & $63.7 \pm 6.0$ & $63.2 \pm 11.9$ \\
BMl $\left(\mathrm{kg} / \mathrm{m}^{2}\right)$ & $27.5 \pm 3.3$ & $26.5 \pm 4.0$
\end{tabular}

Data presented as mean \pm SD 


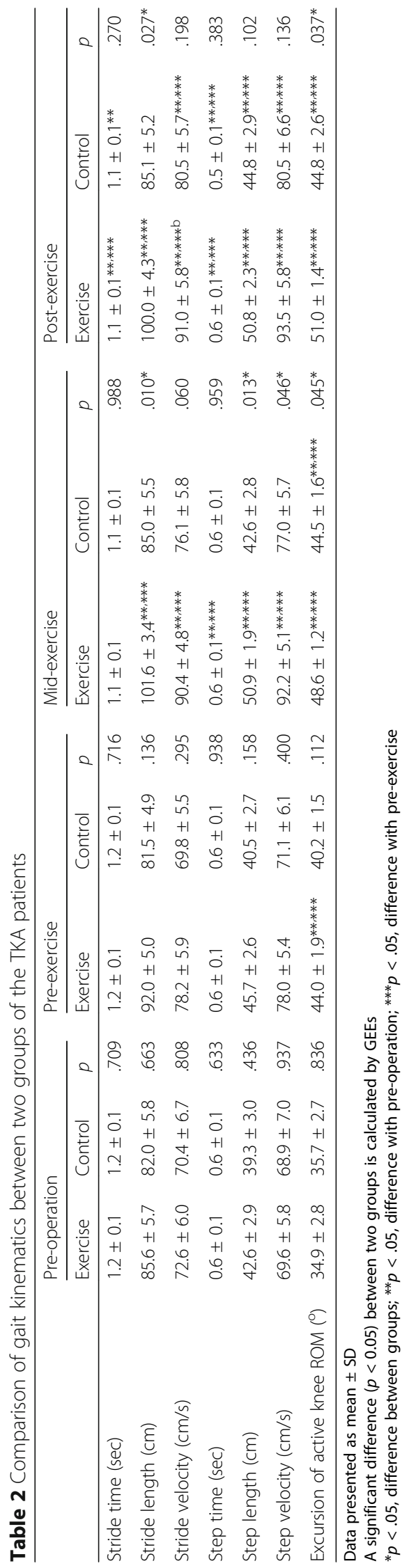




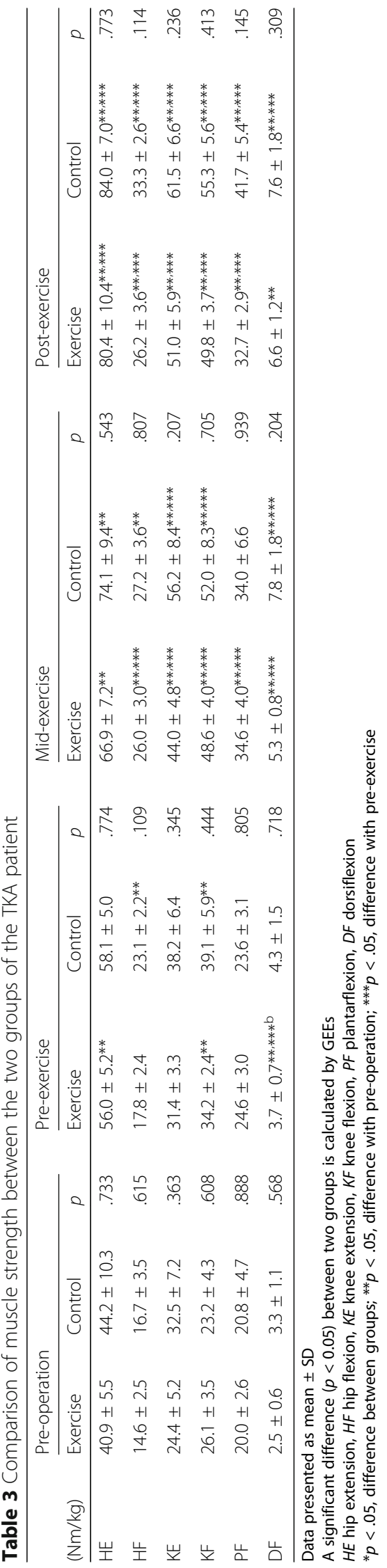


In post-exercise KOOS assessment, KOOS total score was $78.7 \pm 3.2$ and $66.4 \pm 4.1$ for exercise and control group, respectively $(p=0.018)$. In the subcategorical assessment, these differences were observed in pain and ADL $(p<0.05)$ (Table 4$)$. When comparison of KOOS was performed within the individual group, the temporal improvement was observed in symptoms, pain, ADL, QOL, and total score for both exercise and control group $(p<0.05)$ (Table 4$)$. Interestingly, the sports subcategory was shown significantly improved from $17.1 \pm 5.4$ at pre-operative assessment to $48.3 \pm 7.8$ at post-exercise assessment in the exercise group $(p<0.05)$, while no such difference was shown in the control group.

In the SF-36 questionnaire which included physical and mental domains, it was shown a significant increase in the domains of $\mathrm{GH}, \mathrm{SF}$, and PCS at the mid-exercise point in the exercise group compared to the control group $(p<0.05)$ (Table 5). The circuit training efficiently improved the GH, SF, and PCS at the earlier time point. At the post-exercise assessment, only SF and BP were shown a significant increase in the exercise group compared with the control group (Table 5). When comparison was performed within the individual group in temporal fashion, it was shown that exercise improved all mental domains in mid-exercise assessment, and these improvements lasted to the post-exercise assessment, while no such improvement was observed in the control group. In the physical domain, it seemed both groups were shown improvement.

\section{Discussion}

The most significant findings in the present study were that 24-week circuit training resulted in a decrease of pain and an increase of ADL and SF, along with an increase in stride length and excursion of knee ROM in gait analysis [8, 22, 23]. Second, the increases in all muscle strength were demonstrated in a temporal fashion in both exercise and control group [24]. Circuit training seemed not further increase the maximal muscle strength. Third, earlier recoveries in gait parameters, KOOS, and SF-36 at mid-exercise assessment for exercise group were well demonstrated. Although circuit training did not result in a further increase in maximal isokinetic muscle strength, it was postulated to enhance the muscle coordination which facilitated the performance of walking and daily function [25]. The improvement in pain after circuit training both in the KOOS and SF-36 further provided an important basis that knee can undergo unlimited swing and hence to increase the stride length [23].

In gait analysis, stride length and excursion of knee ROM in the exercise group were shown significant increases as compared to control group at the mid- exercise assessment, and these differences lasted to the post-exercise assessment (Table 2). It is well demonstrated that faster restoration of gait patterns in exercise group as compared to the control group. None of the subjects enrolled in the exercise group reported discomfort or injury, and none needed further management. This indicates that our circuit training intervention is safe for TKA patients and feasible to perform following the TKA surgery.

The function of quadriceps strength relied on maximal muscular strength and muscular coordination in TKA patients, which could be improved by post-operative progressive strengthening protocol [24, 26-29]. Quadriceps weakness is a hallmark characteristic of knee osteoarthritis [24, 27, 30, 31]. Post-operatively, quadriceps strength was not comparable to their age-matched counterparts in the mid- or long-term follow-up [29]. Muscle strength recovery in those aging patients underwent TKA followed a temporary pattern [24]. The current study displayed a simultaneous trend in pain and muscle function recovery. Actually, as the pain improved 3 months after TKA, the patient was able to load their knees and hence improve the lower extremity muscle force. As the pain improved more, it was shown that lower leg muscle strength has a corresponding further increase. We have previously demonstrated the progress of both cardiovascular fitness and muscle strength by circuit training and 12-week circuit training in healthy middle-aged women (aged 45-75) [12]. However, the circuit training in the current study did not result in a further increase in the maximal isokinetic muscle strength. Two possibilities existed. First, the intensity of resistant training in the current study could be inadequate and would not result in a further increase as compared to the daily activities [23]. Second, the maximal strength has plateaued. Further study was warranted to delineate the mechanism underlying the findings in the current study. On the other hand, the muscle coordination and performance were improved by circuit training as shown in gait parameters. This finding paralleled literature that modified gait efficacy scale was improved by exercise [23]. In this laboratory study, it was further shown a $15 \%$ increase in stride length, accompanied by $10 \%$ increase in excursion of knee ROM during walking. These results provided a solid basis to explain the positive effect of exercise on gait function. It was possible that the decrease in pain and a more coordinated muscle function improved the gait function.

Decreased exercise adherence usually endangered the effect of exercise intervention [10, 32]. The motives for exercise participation among patients with knee OA was suggested mainly as positive outcome expectation [10]. Other factors such as social interaction and enjoyment of exercise have been described as important facilitators 


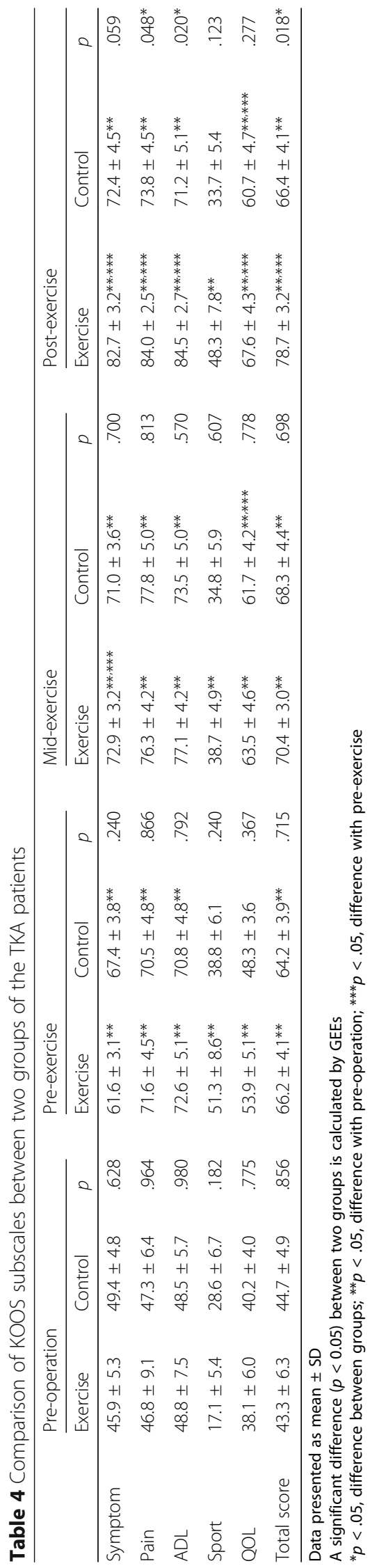


Table 5 Comparison of perceived health (SF-36) between two groups of the TKA patients

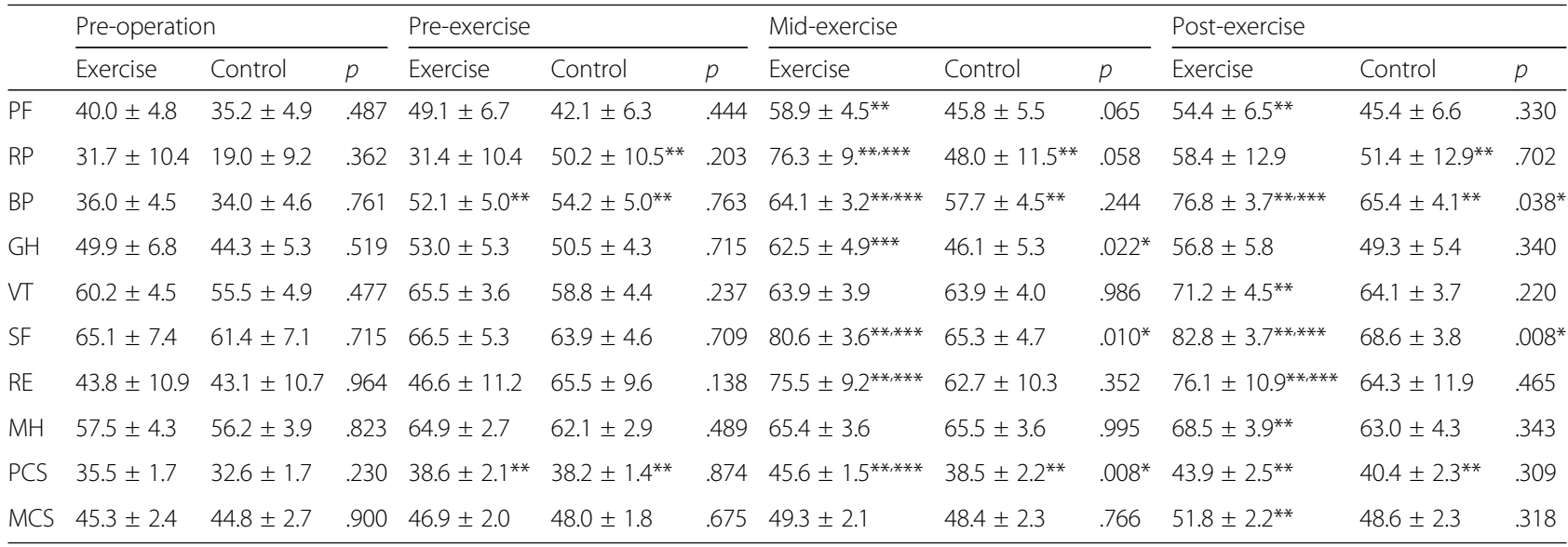

Data presented as mean \pm SD

A significant difference $(p<0.05)$ between two groups is calculated by GEEs

${ }^{*} p<.05$, difference between groups; ${ }^{* *} p<.05$, difference with pre-operation; ${ }^{* * *} p<.05$, difference with pre-exercise

of exercise behavior [22, 32]. The present study demonstrated the improvement in social function which could result in exercise adherence to $80 \%$, which was comparable in OA patient without TKA [10, 22]. Our results supported that a more balanced exercise program in patients with TKA to achieve a better exercise adherence and consequent improvement in gait and function [11]. Furthermore, the present study provided a quantitative data regarding the exercise prescription with efficacy and efficiency, which could be practiced for months. These results could be the basis for developing home exercise regimens.

Several limitations in this study must be acknowledged. First, the small sample size (34 patients) in this study resulted in under power to show a statistical difference between two groups in head-to-head comparisons. However, comparisons in repeated measure provided a better statistical power. Comparisons of repeated measurements in the same patient demonstrated the effect of exercise in a time sequence. Second, the study is limited to the Asian experience of knee arthroplasty. Third, this was a short-term study; thus, we were unable to assess the effect post-operative circuit training in long-term functional outcomes. The resistance training, though might be submaximal, in combination with aerobic exercise and stretching exercise help faster recovery in gait, functional score, and social function. Fourth, this study is a retrospective study. The results of circuit training on TKAs should be further proved by a prospective randomized clinical trial design. Fifth, there was a heterogeneous effect among the patients in the exercise. The previous investigation has shown that the carrier with polymorphism of monocarboxylate transporter 1 gene (A1470T) exhibits a worse lactate transport and influences the performance with high-intensity circuit training [33]. The genetic variance may be responsible for the heterogeneity of circuit training effect on the individual patients. The further study focused on the genetic test before training intervention was suggested.

\section{Conclusions}

Our post-operative circuit training intervention is safe and effective in improving and hastening the functional recovery after TKA surgery, even without enhancement of lower limb muscle strength. This circuit training intervention might be incorporated into the standard post-operative rehabilitation protocol for TKA patients. The ultimate influence of circuit training on TKA needs further long-term investigation.

\section{Abbreviations}

ADL: Activities of daily living; BP: Bodily pain; GEE: Generalized estimating equations; GH: General health; KOOS: Knee injury and Osteoarthritis Outcome Score; MCS: Mental component summary; MH: Mental health; OA: Osteoarthritis; PCS: Physical component summary; PF: Physical functioning; RE: Role Limitation due to emotional problem; ROM: Range of motion; RP: Role Limitation due to physical problems; SF: Social functioning; SF-36: Short-Form Health Survey; VT: Vitality

\section{Acknowledgements}

The authors thank Chao-Ling Lai and Chia-Fang Chang for collecting the participants' data.

\section{Funding}

Financial support from Chang Gung Memorial Hospital Grant CORPG6C002123 is appreciated. There was no external funding.

\section{Availability of data and materials}

The rest of the data are available from the corresponding author upon reasonable request.

\section{Authors' contributions}

WHH and WBH drafted the manuscript and revised it critically for important intellectual content. WHH and WBH contributed equally to the work. SHC performed statistical analysis. WJS, ZRL, and RWWH contributed substantially to the conception and design of the study. All authors read and approved the final manuscript. 


\section{Ethics approval and consent to participate}

This study was approved by the Ethics Committee and Institutional Review Board (IRB:102-0979B) and registered in the ClinicalTrials.gov database (ID: NCT0292856 2). All patients provided informed consent.

\section{Consent for publication}

Not applicable

\section{Competing interests}

The authors declare that they have no competing interests.

\section{Publisher's Note}

Springer Nature remains neutral with regard to jurisdictional claims in published maps and institutional affiliations.

\section{Author details}

'Sports Medicine Center, Chang Gung Memorial Hospital, No. 6, West Section, Chia-Pu Road, Putz City 61363, Chiayi Country, Taiwan, Republic of China. ${ }^{2}$ Department of Orthopaedic Surgery, Chang Gung Memorial Hospital, No. 6, West Section, Chia-Pu Road, Putz City 61363, Chiayi Country, Taiwan, Republic of China. ${ }^{3}$ PO CHENG Orthopedic Institute, No. 100, Bo-ai 2nd Road, Kaohsiung 81357, Zuoying District, Taiwan, Republic of China. ${ }^{4}$ Department of Athletic Sports, National Chung Cheng University, No.168, University Road, Minhsiung Township 62102, Chiayi Country, Taiwan, Republic of China.

\section{Received: 4 August 2017 Accepted: 8 October 2017}

\section{Published online: 19 October 2017}

\section{References}

1. Daigle ME, Weinstein AM, Katz JN, Losina E. The cost-effectiveness of total joint arthroplasty: a systematic review of published literature. Best Pract Res Clin Rheumatol. 2012;26(5):649-58.

2. Ranawat CS, Flynn WF Jr, Saddler S, Hansraj KK, Maynard MJ. Long-term results of the total condylar knee arthroplasty. A 15-year survivorship study. Clin Orthop Relat Res. 1993:286:94-102.

3. Crowninshield RD, Rosenberg AG, Sporer SM. Changing demographics of patients with total joint replacement. Clin Orthop Relat Res. 2006:443:266-72

4. Gillespie GN, Porteous AJ. Obesity and knee arthroplasty. Knee. 2007;14(2):81-6.

5. Kurtz S, Ong K, Lau E, Mowat F, Halpern M. Projections of primary and revision hip and knee arthroplasty in the United States from 2005 to 2030. J Bone Joint Surg Am. 2007;89(4):780-5.

6. Physical Activity Guidelines Advisory Committee Report. 2008.https://health. gov/paguidelines/pdf/paguide.pdf.

7. Pozzi F, Snyder-Mackler L, Zeni J. Physical exercise after knee arthroplasty: a systematic review of controlled trials. Eur J Phys Rehabil Med. 2013;49(6):877-92.

8. Hiyama Y, Kamitani T, Wada O, Mizuno K, Yamada M. Effects of group-based exercise on range of motion, muscle strength, functional ability, and pain during the acute phase after total knee arthroplasty: a controlled clinical trial. J Orthop Sports Phys Ther. 2016;46(9):742-8.

9. Han AS, Nairn L, Harmer AR, Crosbie J, March L, Parker D, Crawford R, Fransen M. Early rehabilitation after total knee replacement surgery: a multicenter, noninferiority, randomized clinical trial comparing a home exercise program with usual outpatient care. Arthritis Care Res (Hoboken). 2015;67(2):196-202.

10. Chen M, Li P, Lin F. Influence of structured telephone follow-up on patient compliance with rehabilitation after total knee arthroplasty. Patient Prefer Adherence. 2016;10:257-64

11. Piva SR, Gil AB, Almeida GJ, DiGioia AM 3rd, Levison TJ, Fitzgerald GK. A balance exercise program appears to improve function for patients with total knee arthroplasty: a randomized clinical trial. Phys Ther. 2010;90(6):880-94.

12. Hsu WH, Hsu RW, Lin ZR, Fan CH. Effects of circuit exercise and tai chi on body composition in middle-aged and older women. Geriatr Gerontol Int. 2015;15(3):282-8.

13. Hame SL, Alexander RA. Knee osteoarthritis in women. Curr Rev Musculoskelet Med. 2013;6(2):182-7.

14. Whitlock KG, Piponov HI, Shah SH, Wang OJ, Gonzalez MH. Gender role in total knee arthroplasty: a retrospective analysis of perioperative outcomes in US patients. J Arthroplast. 2016;31(12):2736-40.

15. Song R, Roberts BL, Lee EO, Lam P, Bae SC. A randomized study of the effects of t'ai chi on muscle strength, bone mineral density, and fear of falling in women with osteoarthritis. J Altern Complement Med. 2010;16(3):227-33.
16. Xie F, Li SC, Roos EM, Fong KY, Lo NN, Yeo SJ, Yang KY, Yeo W, Chong HC, Thumboo J. Cross-cultural adaptation and validation of Singapore English and Chinese versions of the Knee injury and Osteoarthritis Outcome Score (KOOS) in Asians with knee osteoarthritis in Singapore. Osteoarthr Cartil. 2006;14(11):1098-103.

17. Roos EM, Roos HP, Lohmander LS, Ekdahl C, Beynnon BD. Knee injury and Osteoarthritis Outcome Score (KOOS) — development of a self-administered outcome measure. J Orthop Sports Phys Ther. 1998;28(2):88-96.

18. Tsai SY, Chi LY, Lee LS, Chou P. Health-related quality of life among urban rural, and island community elderly in Taiwan. J Formos Med Assoc. 2004; 103(3):196-204

19. K-Y LIANG, ZEGER SL. Longitudinal data analysis using generalized linear models. Biometrika. 1986:73(1):13-22.

20. Maly MR, Costigan PA, Olney SJ. Determinants of self-report outcome measures in people with knee osteoarthritis. Arch Phys Med Rehabil. 2006:87(1):96-104.

21. Yoshida Y, Mizner RL, Ramsey DK, Snyder-Mackler L. Examining outcomes from total knee arthroplasty and the relationship between quadriceps strength and knee function over time. Clin Biomech (Bristol, Avon), 2008;23(3):320-8.

22. Fransen M, McConnell S, Harmer AR, Van der Esch M, Simic M, Bennell KL. Exercise for osteoarthritis of the knee. Cochrane Database Syst Rev. 2015:1:CD004376.

23. Taniguchi M, Sawano S, Kugo M, Maegawa S, Kawasaki T, Ichihashi N. Physical activity promotes gait improvement in patients with total knee arthroplasty. J Arthroplast. 2016;31(5):984-8.

24. Mizner RL, Petterson SC, Snyder-Mackler L. Quadriceps strength and the time course of functional recovery after total knee arthroplasty. J Orthop Sports Phys Ther. 2005;35(7):424-36.

25. Kuntze G, von Tscharner V, Hutchison C, Ronsky JL. Alterations in lower limb multimuscle activation patterns during stair climbing in female total knee arthroplasty patients. J Neurophysiol. 2015;114(5):2718-25.

26. Mizner RL, Petterson SC, Stevens JE, Axe MJ, Snyder-Mackler L. Preoperative quadriceps strength predicts functional ability one year after total knee arthroplasty. J Rheumatol. 2005;32(8):1533-9.

27. Mizner RL, Petterson SC, Stevens JE, Vandenborne K, Snyder-Mackler L. Early quadriceps strength loss after total knee arthroplasty. The contributions of muscle atrophy and failure of voluntary muscle activation. J Bone Joint Surg Am. 2005;87(5):1047-53.

28. Mizner RL, Snyder-Mackler L. Altered loading during walking and sit-tostand is affected by quadriceps weakness after total knee arthroplasty. J Orthop Res. 2005;23(5):1083-90.

29. Ishii Y, Noguchi H, Sato J, Sakurai T. Toyabe SI. Knee Surg Sports Traumatol Arthrosc: Quadriceps strength impairment in the mid- to long-term followup period after total knee arthroplasty; 2016

30. Stevens JE, Mizner RL, Snyder-Mackler L. Quadriceps strength and volitional activation before and after total knee arthroplasty for osteoarthritis. J Orthop Res. 2003;21(5):775-9.

31. Meier W, Mizner RL, Marcus RL, Dibble LE, Peters C, Lastayo PC. Total knee arthroplasty: muscle impairments, functional limitations, and recommended rehabilitation approaches. J Orthop Sports Phys Ther. 2008:38(5):246-56.

32. Bennell K, Dobson F. Review: exercise interventions improve pain and function in people with knee osteoarthritis compared with no exercise. Evid Based Nurs. 2014;17(4):109.

33. Cupeiro R, Benito PJ, Maffulli N, Calderon FJ, Gonzalez-Lamuno D. MCT1 genetic polymorphism influence in high intensity circuit training: a pilot study. J Sci Med Sport. 2010;13(5):526-30 